\title{
La présence conceptuelle du CECR dans les curriculums de français de base du palier secondaire au Canada
}

\author{
Michaël Boucher \\ Université d'Ottawa \\ Marie-Josée Vignola \\ Université d'Ottawa
}

\begin{abstract}
Résumé
En 2001, l'Europe a créé le Cadre Européen Commun de Référence (CECR), en se basant sur l'approche actionnelle et le plurilinguisme, pour encadrer l'apprentissage, l'enseignement et l'évaluation des langues étrangères. Au Canada, le Conseil des ministres de l'Éducation du Canada (CMEC) a souhaité s'inspirer du CECR pour uniformiser les pratiques d'enseignement et d'évaluation des langues secondes (L2). Cet article se penche sur le degré d'intégration de l'approche actionnelle et du plurilinguisme dans les curriculums de français de base (Core French) provinciaux et territoriaux au Canada. Selon le modèle d'analyse documentaire de Van der Maren, nous observons que les curriculums de français de base canadiens intègrent tous des sous-concepts relatifs aux deux concepts du CECR. L'approche actionnelle y est sémantiquement plus représentée que le plurilinguisme.
\end{abstract}

\begin{abstract}
In 2001, the Common European Framework of Reference (CEFR), based on the concepts of an action-oriented approach and multilingualism, was created in Europe to frame the learning, teaching and assessment of foreign languages. In Canada, the Council of the Ministers of Education of Canada (CMEC) drew inspiration from the CEFR to standardize teaching and assessment practices for second language (L2) education. This article examines the degree of integration of the action-oriented approach and plurilingualism in provincial and territorial Core French curricula in Canada. Applying Van der Maren's documentary analysis model, we observe that all Canadian Core French curricula integrate sub-concepts relating to the two CEFR concepts, with the action-oriented approach more represented than plurilingualism.
\end{abstract}




\section{La présence conceptuelle du CECR dans les curriculums de français de base du palier secondaire au Canada}

De nos jours, les technologies de l'information ont transformé la façon dont nous communiquons. De nombreux accords commerciaux ont été signés, ce qui a mené à l'accroissement des mouvements de personnes à travers la planète. L'espace-monde est ainsi plus ouvert qu'auparavant, et les États beaucoup plus perméables aux influences extérieures, notamment culturelles et linguistiques (Le Thiec Rautureau, 2011). Nous évoluons dans une ère mondialisée où l'unilinguisme des nations s'effrite face à ces vents de changements, et où la coexistence de plusieurs langues est désormais monnaie courante (Conseil de 1'Europe, 2001). Dorénavant, les sociétés doivent s'adapter à ce monde plus complexe (Piccardo, 2013b). Il y a ainsi davantage de rencontres interculturelles et interlinguistiques, desquelles ont émergé de nouveaux besoins communicationnels entre personnes aux bagages culturel et linguistique plus diversifiés. C'est dans ce contexte davantage hétéroclite que les acteurs du milieu de l'enseignement des langues étrangères ont réfléchi à la conception d'un outil pédagogique plus adapté à cette nouvelle complexité sociolinguistique.

Dans la lignée des recherches de North (2000) quant à l'élaboration d'un cadre commun en matière de maîtrise des langues étrangères, le Conseil de l'Europe $(\mathrm{CoE}) \mathrm{a}$ élaboré en 2001 le Cadre européen commun de référence pour les langues aussi appelé CECR (Conseil de l'Europe, 2001). Le but était avant tout de mettre en place des bases communes pour l'apprentissage, l'enseignement et l'évaluation des langues étrangères, puis de favoriser les échanges linguistiques et culturels à l'échelle des pays du CoE (et audelà), et de renforcer le concept de communauté européenne (Le Thiec Rautureau, 2011). Pour le CoE, l'objectif était donc, au départ, de faire passer le citoyen européen d'un statut unilingue à plurilingue. Le CECR répondait directement à de nouveaux besoins communicationnels interindividuels sur les plans linguistique et culturel, puisqu'il était essentiel d'établir une cohérence et une uniformité des pratiques d'enseignement et d'évaluation des langues étrangères (Conseil de l'Europe, 2001).

Près d'une décennie plus tard, Little et Taylor (2013) affirment que le CECR offre effectivement une base commune pour l'apprentissage, l'enseignement et l'évaluation des langues étrangères. Le but premier du CECR est de développer des compétences langagières sur les plans linguistique, culturel, mais aussi pragmatique, c'est-à-dire correspondant à l'utilisation de la langue cible en contexte social (Le Thiec Rautureau, 2011). Pour assurer le développement de ces compétences, les architectes du CECR ont élaboré des descripteurs de tâches, soit de courts énoncés décrivant les objectifs et les modalités des diverses tâches sociales à accomplir par les apprenants. Le CECR tire une partie de son architecture conceptuelle de l'approche actionnelle, selon laquelle l'apprenant d'une langue est avant tout un acteur social interagissant avec d'autres membres de la communauté (Conseil de l'Europe, 2001). Le développement des compétences langagières s'opère donc par l'entremise de simulation de tâches sociales complexes (Conseil de 1'Europe, 2001).

Le CECR se distingue aussi par sa flexibilité d'application, et ne se présente pas telle une méthode d'enseignement standardisée. Il s'agit plutôt de principes directeurs flexibles, dans la mesure où le CECR a été créé pour répondre aux changements sociolinguistiques amenés par la mondialisation, le monde n'étant plus unilingue, mais 
bien plurilingue. En effet, les individus de différents pays sont de plus en plus en interaction les uns avec les autres, et c'est dans ce contexte que le CECR a dépassé depuis 2001 les frontières de l'Europe, pour atteindre d'autres régions du monde dont le Canada (Martyniuk et Noijons, 2007; Piccardo 2013b, 2016b).

Au Canada, c'est tout d'abord Vandergrift (2006) qui a introduit le CECR et qui a partagé ses nombreux avantages pédagogiques pour l'apprentissage, l'enseignement et l'évaluation des langues étrangères ou secondes (L2). Il a de plus avancé que la mobilité des Canadiens au sein de la fédération canadienne requérait d'avoir une terminologie commune pour la définition d'une communication efficace dans l'apprentissage d'une L2. Puis, s'inspirant des principales suggestions de Vandergrift, le Conseil des ministres de l'Éducation du Canada (CMEC) a publié en 2010 un guide à l'intention des provinces et des territoires pour les inciter à adopter le CECR, et ce, de manière à uniformiser à l'échelle nationale les pratiques d'enseignement et d'évaluation des L2 pour favoriser les apprentissages en la matière. C'est d'ailleurs la première fois qu'une instance fédérale se prononçait en faveur d'un outil pédagogique en didactique des langues commun à toutes les provinces (Faez et coll., 2011; Piccardo, 2013b). Mentionnons par ailleurs que l'éducation au Canada relève des gouvernements provinciaux. Par conséquent, il en va donc de la responsabilité de chaque province de suivre, ou non, ces recommandations.

Ainsi, depuis la parution du guide du CMEC (2010), maints projets pilotes, visant à mettre en place des pratiques pédagogiques inspirées du CECR, ont vu le jour au Canada. À titre d'exemple, des études menées dans des classes de français de base (Core French) en Ontario ont montré que l'introduction du CECR améliorait la motivation des élèves, car ceux-ci utilisaient la langue en contexte de communication réelle (Faez et coll., 2011). Par conséquent, les élèves développaient davantage leur confiance, et étaient plus autonomes dans la réalisation de leur apprentissage linguistique. Le CECR a ici eu un impact positif, notamment car les élèves s'appuyaient davantage sur leurs propres expériences (Faez et coll., 2011). Par ailleurs, le CECR encourage grandement l'utilisation de documents authentiques, ce qui a également contribué à rehausser la motivation des élèves à apprendre le français langue seconde (FLS) (Vandergrift, 2015). Comme le CECR définit les élèves en tant qu'acteurs sociaux, ceux-ci étaient également en mesure de critiquer et d'évaluer leurs propres apprentissages, ce qui, combiné au caractère réel et authentique des tâches, augmentait leur motivation à utiliser la langue cible en classe (Faez et coll., 2011). Ainsi, après avoir passé en revue quelques exemples des retombées pédagogiques d'une pratique influencée du CECR, nous aborderons dans la partie suivante l'utilisation du CECR à l'échelle pancanadienne.

Ainsi, dix ans après la parution du guide du CMEC, nous avons observé qu'il est plutôt ardu d'avoir un portrait clair de l'intégration du CECR dans les provinces et les territoires au Canada. Cela s'explique par le fait que la recherche reste lacunaire quant à son application en contexte scolaire de la maternelle à la $12^{\mathrm{e}}$ année, et qu'elle met souvent trop l'accent sur l'Europe et non sur le Canada (Arnott et coll., 2017). Cet état de fait est problématique, dans la mesure où $75 \%$ des élèves qui fréquentent les écoles de langue anglaise au Canada sont inscrits dans des programmes de français de base (Canadien Parents for French, 2019). Il serait donc bénéfique au Canada d'établir des critères communs dans l'apprentissage, l'enseignement, et l'évaluation du FLS. À ce titre, le Comité permanent des langues officielles de la Chambre des communes a déposé en 2007 un rapport recommandant l'établissement d'un cadre canadien commun de référence 
influencé par le CECR, dans le but d'améliorer les programmes de FLS partout au Canada, ce qui, rappelons-le, correspond à la mission fondamentale et première du CECR, soit celle d'établir un cadre supranational et de favoriser la coopération entre pays en ce qui a trait à l'enseignement des langues étrangères (Comité permanent des langues officielles, 2017). Ainsi, l'établissement d'un tel cadre bénéficierait à tous les élèves canadiens de FLS, dans la mesure où bon nombre d'entre eux poursuivent leur scolarité dans d'autres provinces et territoires (CMEC, 2015).

C'est dans cette optique qu'il nous semble opportun et nécessaire d'effectuer une analyse descriptive et comparative des curriculums provinciaux et territoriaux en nous concentrant de façon particulière sur le français de base au palier secondaire, puisque tous les territoires (à l'exception du Nunavut) et provinces offrent le français de base, de la $9^{\mathrm{e}}$ à la $12^{\mathrm{e}}$ année (et de la première à la cinquième année du secondaire au Québec). Il est d'ailleurs pertinent d'effectuer une analyse descriptive des curriculums de français de base, puisque la recherche en FLS au Canada a tendance à se concentrer plus souvent sur l'immersion française. Ainsi, des recherches ont notamment été menées sur le plurilinguisme en lien avec le FLS au Canada, mais plus souvent en contexte d'immersion française (Dagenais, 2008). Plus spécifiquement, il est question, dans le projet de recherche qui fait l'objet de cet article, d'observer si les pratiques en matière d'enseignement et d'évaluation du français de base des provinces et territoires sont en adéquation avec les recommandations du CMEC visant l'intégration du CECR. Cet article a donc pour but de présenter le niveau d'intégration du CECR dans les curriculums de français de base des provinces et territoires canadiens, et ce, en se penchant plus précisément sur les deux principaux concepts le constituant, soit l'approche actionnelle et le plurilinguisme. Pour ce faire, nous nous sommes attelés à détecter, dans tous les curriculums de français de base des provinces et des territoires, les traces des deux concepts constitutifs du CECR.

\section{Cadre conceptuel et recension des écrits}

Penchons-nous dès lors sur les articles ayant guidé la réalisation de ce projet de recherche. Ainsi, les écrits sélectionnés pour la recension des écrits et le cadre conceptuel font part dans un premier temps de l'influence du CECR en contexte canadien, puis, dans un deuxième temps, abordent plus en profondeur les deux concepts fondamentaux que sont l'approche actionnelle et le plurilinguisme.

\section{Cadre européen commun de référence en contexte canadien}

Tel que susmentionné, c'est Vandergrift qui, en 2006, a introduit cet outil pédagogique qu'est le CECR au Canada. C'était la première fois qu'une volonté claire d'harmoniser les méthodes d'enseignement et d'évaluation des L2 était énoncée au pays. Vandergrift souligne d'emblée qu'aucun instrument de mesure des apprentissages des élèves dans leur L2 n'avait jusque-là existé au Canada. À cet effet, les descripteurs de tâches s'avèrent utiles pour les élèves, qui peuvent ainsi mesurer leurs apprentissages, rehaussant du même coup leur motivation et leur autonomie (Faez et coll., 2011). De plus, selon Vandergrift, les provinces et territoires seraient en mesure d'améliorer leurs pratiques d'enseignement et d'évaluation, en partageant une vision pancanadienne harmonisée de la définition d'une compétence langagière. Pour Vandergrift, le CECR est valide, car il a été 
préalablement testé, empiriquement et authentiquement, et ce, au sein de maints contextes pédagogiques. Ses descripteurs ont été élaborés à partir de l'expérience même des enseignants et des apprenants de langue étrangère en Europe. De plus, cet outil est modulable en fonction des différents besoins des provinces et territoires canadiens (Vandergrift, 2016). Pour lui, le CECR offre donc aux provinces et territoires la chance de partager une terminologie commune basée sur les tâches sociales et les compétences langagières à développer. Ici, le caractère authentique du CECR fait en sorte que l'élève en vient à construire ses apprentissages, non seulement par l'interaction avec ses pairs, mais également avec celle des locuteurs de la langue cible (Piccardo, 2016b; Vandergrift, 2016). Vandergrift a donc été le premier au Canada à vanter les mérites pédagogiques du CECR, notamment car cet outil présente l'élève comme un acteur social, tel que nous le verrons dans la partie subséquente portant sur l'approche actionnelle, un des piliers conceptuels du CECR.

Par ailleurs, mentionnons également que le CECR a depuis 2001 été peaufiné à maintes reprises. La toute nouvelle version, publiée en 2020, accorde une plus grande part à l'approche actionnelle et au plurilinguisme, tout comme à la médiation. En outre, une approche plus transdisciplinaire de l'enseignement des langues a aussi influencé davantage les lignes directrices du document. Il appert aussi pertinent de mentionner que de nombreux enseignants canadiens ont aussi participé à la phase de validation, témoignant ainsi d'une volonté d'internationaliser les horizons géographiques de l'outil. Cela étant établi, attardons-nous maintenant à l'un des deux concepts d'importance du CECR, soit l'approche actionnelle.

\section{Approche actionnelle}

À la lumière du complément publié en 2020, il est clair que l'approche actionnelle structure une bonne partie des grands pans didactiques du CECR (2020). Ainsi, pour l'approche actionnelle, l'objectif est pour l'élève de communiquer ses intentions en contexte social. Pour ce faire, le CECR l'amène à réaliser des tâches sociales en utilisant tout l'éventail de ses compétences langagières, soit linguistiques (connaissance de la langue, de son lexique, de sa morphosyntaxe et de sa phonétique), sociolinguistiques (normes sociales influençant les paramètres d'usage de la L2) et pragmatiques (choix discursifs visant la compréhension de l'intention du discours, soit en l'adaptant, l'organisant et le structurant en fonction des locuteurs présents dans la situation de communication) (Conseil de l'Europe, 2001). Une tâche n'est complète que lorsque toutes ces compétences langagières sont prises en compte dans une même situation. Dans ce casci, l'élève utilise la langue pour communiquer et pour accomplir des tâches sociales complexes et authentiques en lien avec son quotidien (Rosen, 2010). Par exemple, dans un contexte canadien, un élève de FLS pourrait discuter avec d'autres apprenants de la nécessité de permettre ou non les bagarres au hockey. L'utilisation du français s'inscrit donc hors du cadre formel d'enseignement, reflétant plutôt la société dans laquelle les élèves évoluent au quotidien (Le Thiec Rautureau, 2011). L'approche actionnelle agit ainsi comme une simulation de la vie réelle des apprenants (Piccardo, 2016a; Piccardo et North, 2019). La rétroaction entre pairs est aussi importante, car elle reflète la façon dont les acteurs sociaux interagissent. Ainsi, ces compétences langagières sont utiles « dans et pour l'action sociale » (Piccardo, 2016a, p.68). 
Sur le plan de l'évaluation, les compétences langagières caractérisant l'approche actionnelle s'avèrent aussi efficaces pour l'autoévaluation de l'élève, puisqu'elles sont articulées sous la forme de descripteurs clairs (Little et Taylor, 2013). Ainsi, l'apprenant peut ultérieurement effectuer des retours pour améliorer certaines facettes de ses apprentissages lors d'une tâche donnée. Il en vient donc par le fait même à développer sa métacognition (Little et Taylor, 2013).

Malgré les avantages d'une pédagogie basée sur l'approche actionnelle, Little et Taylor (2013) font remarquer que la recherche au Canada se concentre trop sur des problématiques théoriques qui, bien souvent, ne sont pas pertinentes au contexte canadien. Ils soulignent aussi que de nombreux enseignants sont réticents à l'idée de modifier leurs pratiques d'enseignement et d'évaluation du FLS pour s'inspirer davantage de l'approche actionnelle, et ce, même si des études exploratoires ont fait état de son potentiel pédagogique, notamment en ce qui concerne un usage accru de la langue cible en classe (Little et Taylor, 2013). Piccardo (2013a) abonde dans le même sens, en avançant que les enseignants observent bel et bien la valeur pédagogique de l'approche actionnelle et du CECR, mais qu'ils la considèrent trop difficile à mettre en pratique. Par ailleurs, l'absence de formation continue et de soutien aux enseignants n'aide en rien (Faez et coll., 2011; Piccardo, 2013a). D'ailleurs, de telles réticences de la part des enseignants avaient également été observées en Europe avant l'adoption du CECR en 2001 (Little et Taylor, 2013).

Little et Taylor (2013) font aussi mention des critiques à l'encontre des descripteurs de tâches, notamment quant à leur manque de spécificité. Toutefois, ils affirment que cela est dû au fait qu'ils doivent être adaptés en fonction des différents contextes pédagogiques et nationaux. De pair avec Piccardo (2013a), ils mettent aussi de l'avant que l'intégration du CECR, et de l'approche actionnelle, doit s'inscrire dans la longue durée, vu la complexité de l'outil.

En ce qui a trait à l'introduction du CECR et de l'approche actionnelle au Canada, Arnott et coll. (2017) affirment d'emblée que cet outil viendrait combler beaucoup de lacunes dans l'apprentissage, l'enseignement et l'évaluation du FLS au Canada. Il est question, entre autres, de la sévère attrition dans les classes de français de base, découlant d'une désillusion à l'égard des résultats concrets d'apprentissages (Faez et coll., 2011; Lapkin et coll., 2009). L'approche actionnelle viendrait ainsi pallier en partie cette lacune, notamment car elle simule la vie réelle des élèves, ce qui peut augmenter leur motivation. La langue cible devient donc un outil pour développer les compétences langagières, et non un objet d'apprentissage déconnecté. Cela dit, l'élève est loin de n'être qu'un acteur social. Il est aussi porteur d'un bagage linguistique et culturel qui lui est propre. C'est donc dans cette optique que le plurilinguisme constitue, lui aussi, un pilier conceptuel du CECR.

\section{Plurilinguisme}

Le plurilinguisme représente le second pilier conceptuel constitutif du CECR. Dans cette optique plurielle, il est en effet nécessaire en L2 de tenir compte du bagage linguistique et culturel de l'apprenant pour réaliser des apprentissages. Moore et Castellotti (2008) mettent de l'avant que : « dans les sociétés contemporaines, la quasi-totalité des locuteurs dispose d'un répertoire plurilingue, c'est-à-dire d'un ensemble de ressources plurielles qui se combinent d'une manière déséquilibrée et évolutive » (p. 24). Vandergrift 
(2006) a également fait mention du besoin de tenir compte du bagage linguistique et culturel de plus en plus diversifié des élèves canadiens. Il s'agit ici de s'adapter à cette nouvelle complexité sociolinguistique. En outre, Piccardo (2016a) affirme que le plurilinguisme s'arrime aussi parfaitement au contexte global mondialisé. Ce concept se définit comme suit :

Dans le plurilinguisme [...] le principe de relation est central : au fur et à mesure que l'expérience langagière d'un individu dans son contexte culturel s'étend de la langue familiale à celle du groupe social puis à celle d'autres groupes (que ce soit par apprentissage scolaire ou sur le tas), il/elle ne classe pas ces langues et ces cultures dans des compartiments séparés, mais construit plutôt une compétence communicative à laquelle contribuent toute connaissance et toute expérience des langues et dans laquelle les langues sont en corrélation et interagissent. Dans des situations différentes, un locuteur peut faire appel avec souplesse aux différentes parties de cette compétence pour entrer efficacement en communication avec un interlocuteur donné. (Conseil de l'Europe, 2001, p.11)

Pour Moore et Castellotti (2008), la compétence plurilingue en est une unique à chaque élève qui est, grâce à celle-ci, capable d'exprimer son identité socioculturelle au sein d'un contexte social particulier. La communication entre individus aux bagages divers s'inscrit désormais comme une donne fondamentale de notre époque mondialisée. Pour être réussie, cette communication doit tenir compte des particularités linguistiques et culturelles de chacun. La compétence plurilingue établit donc des ponts communicatifs entre les individus, les langues, les cultures et les sociétés (Moore et Castellotti, 2008). Un élève plurilingue est apte, de surcroît, à s'orienter dans cet univers sociolinguistique complexe. Cela lui permet de mieux appréhender les dynamiques linguistiques et culturelles propres à son contexte (Moore et Castellotti, 2008). Puisque l'élève est considéré comme un acteur social, le plurilinguisme s'avère par ailleurs pertinent dans la mesure où les contextes langagiers se sont aujourd'hui grandement complexifiés (Piccardo, 2016a).

Avec le plurilinguisme, l'univers pédagogique s'ouvre dorénavant davantage. La classe de FLS devient de facto plus flexible et plus dynamique. Elle permet aux élèves de faire preuve de plus de créativité et, ainsi, d'élargir les perspectives d'apprentissage audelà des quatre murs de la classe, étant donné que l'apprentissage ne se limite pas qu'à celui de la langue cible (Piccardo, 2016a). L'apprentissage d'une L2 n'est donc plus un processus linéaire, mais devient plutôt interactif et itératif (Piccardo, 2017). Par l'entremise du plurilinguisme, le CECR établit ainsi une synergie entre l'élève et la langue cible. Au Canada, cette synergie s'avère salutaire vu le multiculturalisme caractérisant la démographie des classes (Vandergrift, 2006). De cette façon, les élèves canadiens inscrits en FLS seraient davantage en mesure de s'appuyer sur leur propre « parcours de constructions des connaissances » (Piccardo, p. 68). Finalement, les élèves ne sont plus uniquement des acteurs sociaux, mais aussi des individus à la fois plurilingues et pluriculturels (Moore et Castellotti, 2008). Ayant ainsi abordé toute la complexité sociale, culturelle et linguistique de l'élève, il convient dès lors de se pencher plus précisément sur les visées globales et précises de notre projet de recherche. 


\section{Objectif global et question de recherche}

À l'instar de Vandergrift (2006), Arnott et coll. (2017) recommandent eux aussi que le CECR soit intégré systématiquement dans les pratiques d'enseignement et d'évaluation du FLS au Canada, de la maternelle à la $12^{\mathrm{e}}$ année, et ce, à dessein d'améliorer les résultats d'apprentissage en ce sens. Ces mêmes auteurs mettent également de l'avant l'enthousiasme dont ont fait preuve les enseignants à qui l'on a présenté le CECR, et, de façon plus spécifique, l'approche actionnelle et le plurilinguisme (Arnott et coll., 2017). Ces chercheurs avancent en outre que le CECR complémente l'approche communicative en ce qui concerne l'enseignement et l'évaluation des L2 au Canada (Arnott et coll., 2017). Dans leur recherche, ils ont également constaté que les élèvent utilisaient davantage la langue cible dans une classe inspirée des préceptes du CECR, et qu'ils évaluaient par ailleurs constamment toutes les étapes de leurs apprentissages par l'entremise de l'interaction et de la communication orales (Arnott et coll., 2017). Ainsi donc, ils suggèrent qu'il serait pertinent d'effectuer une analyse de l'ensemble des programmes

d'enseignement des L2 au Canada, incluant le FLS, pour y détecter les traces conceptuelles du CECR à travers l'approche actionnelle et le plurilinguisme. Selon eux, cela contribuerait ainsi à améliorer, à coordonner et à uniformiser les pratiques d'enseignement et d'évaluation du FLS, notamment du français de base, au Canada (Arnott et coll., 2017).

Nous remarquons néanmoins que la recherche au sujet de l'implantation du CECR dans les curriculums de français de base reste lacunaire, ce qui rend difficile de brosser un portrait global clair de la situation. Certes, quelques études exploratoires ont été menées auprès du corps enseignant quant à l'introduction du CECR en classe. Néanmoins, il a été récemment montré, dans une étude comparative entre la Suisse et le Canada, que l'introduction de nouveaux outils pédagogiques en enseignement des L2, comme le CECR, s'inscrit inévitablement dans la longue durée (Piccardo et coll., 2019). Il demeure aussi toutefois difficile de savoir si les différents ministères de l'Éducation provinciaux et territoriaux ont bel et bien mis en place les recommandations émises par le CMEC en 2010. C'est ainsi qu'une analyse descriptive des curriculums de français de base des provinces et des territoires canadiens du palier secondaire s'avère des plus pertinentes, avec pour objectif de déterminer si l'essence conceptuelle du CECR en matière d'apprentissage, d'enseignement et d'évaluation du français de base y a été, ou non, introduite. L'objectif global du projet de recherche que nous avons mené a donc été d'étudier en détail tous les curriculums de français de base du palier secondaire des provinces et territoires canadiens pour mesurer l'étendue de l'intégration de l'approche actionnelle et du plurilinguisme. Cela va ainsi de pair avec les recommandations émises par le CMEC en 2010 à ce sujet. Pour ce faire, nous nous sommes penchés plus précisément sur la présence, dans chaque curriculum, des sous-concepts de l'approche actionnelle et du plurilinguisme. La question de recherche a donc été formulée de la manière suivante :

Quel est le degré d'intégration des sous-concepts de l'approche actionnelle et du plurilinguisme, concepts constitutifs du CECR, dans les curriculums de français de base du palier secondaire des dix provinces et des trois territoires canadiens?

Cette question vient circonscrire le sujet de recherche dans le décor de l'enseignement du français de base au Canada, et ce, dans un contexte de plus en plus 
hétéroclite sur le plan sociolinguistique, compte tenu de la mondialisation qui remet en question les paramètres définissant l'apprentissage authentique d'une L2. Cela étant établi, poursuivons maintenant avec la méthodologie qui a rendu possible l'opérationnalisation des différentes étapes de cette recherche.

\section{Méthodologie}

Cette recherche est de type qualitatif et exploratoire (Creswell, 2019), puisqu'elle effectue un tour d'horizon des curriculums de français de base des provinces et territoires canadiens du palier secondaire pour y déceler le niveau d'intégration des sous-concepts de l'approche actionnelle et du plurilinguisme. Elle s'articule selon le modèle de Van der Maren (2004) de l'analyse de contenu. En somme, il s'agit de se pencher sur le contenu de documents ministériels dont les données sont manifestes, étant donné que leur sens est interprété tel quel (L'Écuyer, 1987). Cela étant déterminé, penchons-nous maintenant sur toutes les composantes de la méthodologie sur laquelle cette recherche est basée, soit le corpus, l'instrument d'analyse et l'analyse de contenu.

\section{Corpus}

Les documents sélectionnés pour cette recherche se composent de tous les curriculums de français de base des provinces et territoires canadiens du palier secondaire, de la $9^{\mathrm{e}}$ à la $12^{\mathrm{e}}$ année, à l'exception du Québec dont la scolarité obligatoire se termine à la cinquième secondaire (l'équivalent de la $11^{\mathrm{e}}$ année pour les autres provinces canadiennes). Ce palier a été choisi, car tous les territoires et provinces y offrent le cours de français de base, de la $9^{\mathrm{e}}$ à la $12^{\mathrm{e}}$ année. Voici donc les curriculums provinciaux qui ont servi à l'analyse de cette recherche, et qui étaient publiés au moment de la cueillette de données. Ils apparaissent dans le Tableau 1 selon l'ordre chronologique de leur année de publication. Ce tableau contient trois colonnes, soit pour l'année de publication, la province ainsi que l'acronyme correspondant à la province et qui sera utilisé dans la présentation des résultats. Par ailleurs, il est à noter que chacun des deux curriculums territoriaux correspond à celui de la province adjacente. Par conséquent, le Yukon utilise le curriculum de français de base de la Colombie-Britannique, et les Territoire-du-Nord-Ouest celui de l'Alberta. Rappelons qu'il n'y a pas de curriculum de français de base au Nunavut, puisque le français de base n'y est pas offert. 


\section{Tableau 1}

Liste des curriculums de français de base provinciaux au Canada par ordre chronologique de publication

\begin{tabular}{lll}
\hline Année de publication & Province & Acronyme \\
\hline 1998 & Saskatchewan & SASK \\
\hline 2000 & Île-du-Prince-Édouard & I-P-É \\
\hline 2003 & Nouvelle-Écosse & N-É \\
\hline 2004 & Alberta & ALB \\
\hline 2005 & Québec & QC \\
\hline 2013 & Terre-Neuve-et-Labrador & T-N-L \\
\hline 2014 & Ontario & ONT \\
\hline 2014 & Manitoba & MAN \\
\hline 2016 & Nouveau-Brunswick & N-B \\
\hline 2018 & Colombie-Britannique & C-B \\
\hline
\end{tabular}

Poursuivons avec la Figure 1 qui nous offre un aperçu global des dates de publication pour chaque province et territoire. Cela s'avère utile dans la mesure où nous pouvons apercevoir dans une perspective pancanadienne l'évolution chronologique de la publication de l'ensemble des curriculums provinciaux.

\section{Figure 1}

Carte pancanadienne des dates de publications des curriculums de français de base

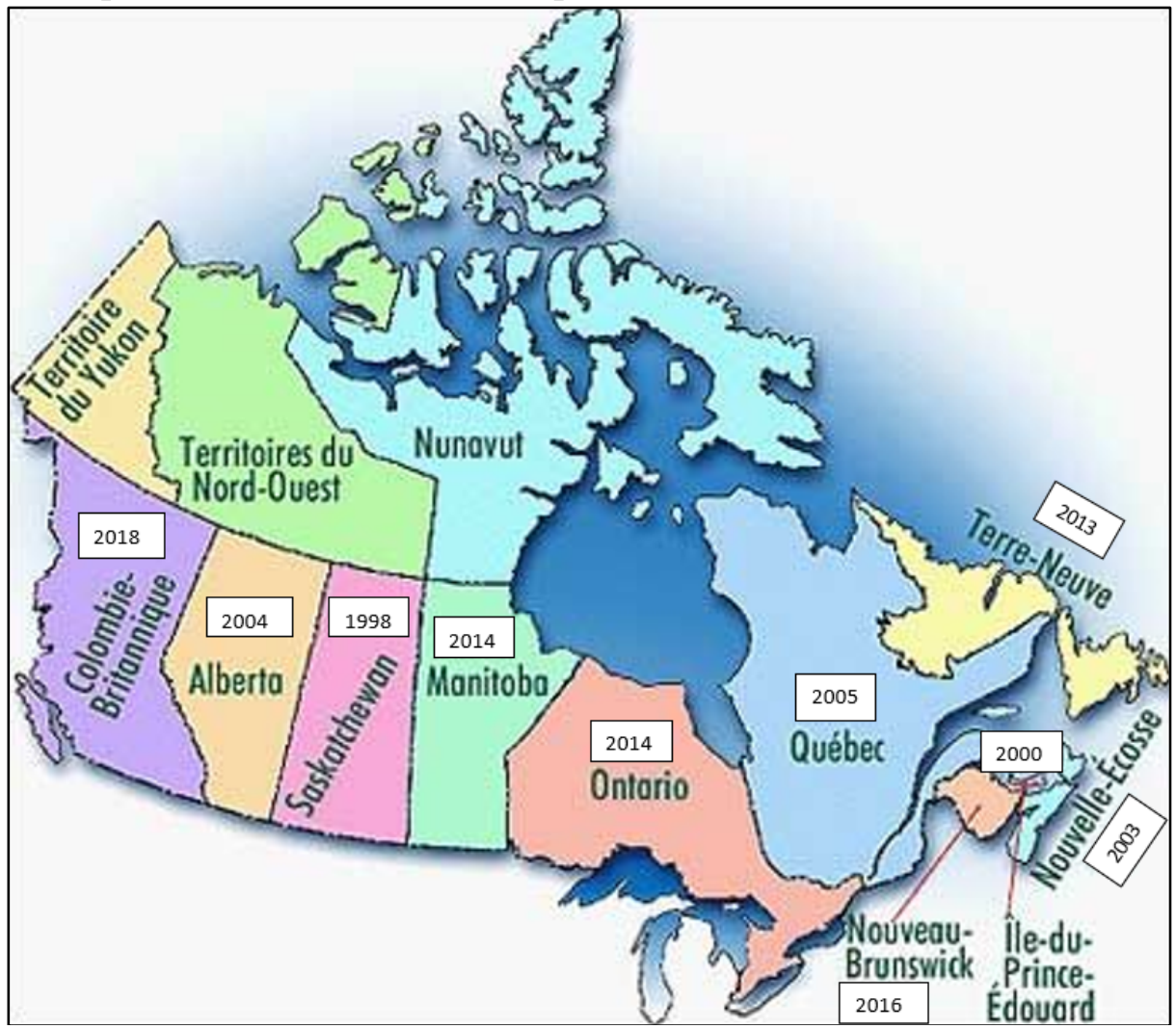

Notons que trois curriculums ont été publiés en français (Île-du-Prince-Édouard, Nouvelle-Écosse et Québec) et sept en anglais (Terre-Neuve-et-Labrador, Nouveau- 
Brunswick, Ontario, Manitoba, Saskatchewan, Alberta et Colombie-Britannique). Le corpus utilisé pour l'analyse comprend les sections expliquant les orientations conceptuelles de chacun de ces curriculums. Ces dernières revêtent un intérêt capital, car elles véhiculent les priorités pédagogiques, leur contenu étant édicté par les décideurs provinciaux et territoriaux (Reis et $\mathrm{Ng}-\mathrm{A}-F o o k, 2010)$ :

Curriculum documents detail the knowledge and skills that students are expected to develop in each subject at each grade level as part of an effort by the government (local or federal) to set standards for schools... curricula are political texts, and as such they result from tensions among a myriad of social, political, and economic forces and movements, which battle to decide what knowledge is of most worth. (p.1015)

Cet exercice nous a donc permis d'observer si les différents ministères de l'Éducation avaient intégré les recommandations du CMEC (2010) en lien avec le CECR. C'est donc de la volonté des acteurs de ce milieu dont il est ici question, étant donné que le CMEC a publié le guide à l'intention des provinces et des territoires il y a déjà 10 ans. Poursuivons maintenant avec la présentation de l'instrument d'analyse.

\section{Instrument d'analyse}

Pour mener à bien cette recherche, nous avons conçu une grille d'extraction divisée en deux parties correspondant à l'approche actionnelle et au plurilinguisme (voir Annexe A). Chacune de ces parties est subdivisée en sous-concepts, tous tirés des définitions de l'approche actionnelle et du plurilinguisme provenant de la version la plus récente du CECR (Conseil de l'Europe, 2018). Dans la colonne de gauche de cette grille, celle des occurrences, est ainsi comptabilisé le nombre de mentions explicites relatives à chaque sous-concept. Puis, dans la colonne de droite, sont pour leur part calculés les pourcentages de l'occurrence de chaque sous-concept par rapport au nombre total d'occurrences repérées dans le curriculum. Par ailleurs, l'ordre des sous-concepts dans la grille reflète l'interprétation du chercheur à l'égard du contenu des divers curriculums (Karsenti et Savoie-Zajc, 2011). Penchons-nous maintenant sur les sous-concepts relatifs à l'approche actionnelle et au plurilinguisme (voir Annexe B). Débutons dans un premier temps avec le Tableau 2 présentant l'ensemble des sous-concepts constitutifs de l'approche actionnelle. 
Tableau 2

Sous-concepts de l'approche actionnelle

Acteur social

Contexte social

Tâches

Besoins communicatifs

Stratégies d'apprentissage

Utilisateur

Collaboration et co-construction

Métacognition

Interaction et communications orales

Médiation

Compétence langagière linguistique

Compétence langagière pragmatique

Compétence langagière sociolinguistique

Maintenant que les sous-concepts constituant l'approche actionnelle ont été présentés, il est dès lors de mise d'enchainer avec le Tableau 3 exposant les sous-concepts composant le plurilinguisme.

Tableau 3

Sous-concepts du plurilinguisme

Répertoire pluriculturel

Répertoire plurilingue

Compréhension plurilingue

Médiateur interculturel

Dans cette section, il a donc été question de deux éléments fondamentaux de la méthodologie constitutive de ce projet de recherche. Ainsi, nous avons présenté le corpus à l'étude de même que l'instrument d'analyse, soit la grille d'extraction ayant rendu possible l'opérationnalisation de l'extraction des données des curriculums. Cela étant établi, attardons-nous plus précisément à l'analyse de contenu, section dans laquelle nous ferons état des différentes étapes méthodologiques propres à ce projet de recherche.

\section{Analyse de contenu}

Van der Maren (2004) divise l'analyse de contenu en trois étapes, soit l'extraction des données, leur examen et leur traitement. Ce sont ces étapes que nous avons suivies pour l'analyse du contenu des curriculums. Le Tableau 4 applique les procédures de Van der Maren à notre sujet de recherche, soit le niveau d'intégration conceptuel de l'approche actionnelle et du plurilinguisme dans les curriculums de français de base du palier secondaire des provinces et territoires au Canada. 


\section{Tableau 4}

Analyse de contenu des curriculums provinciaux et territoriaux de français de base au Canada : Les sous-concepts relatifs à l'approche actionnelle et au plurilinguisme du CECR (Conseil de l'Europe, 2001, 2018) selon le modèle de Van der Maren (2004)

$\begin{array}{ll}\begin{array}{l}\text { Étape 1 : } \\ \text { Extraction des données }\end{array} & \begin{array}{l}\text { Extraire les données des curriculums en s'appuyant sur une liste de codes } \\ \text { tirés des termes définissant les sous-concepts de l'approche actionnelle } \\ \text { et du plurilinguisme tels que définis dans les documents officiels du } \\ \text { CECR. }\end{array} \\ \begin{array}{ll}\text { Étape 2 : } \\ \text { Examen des données }\end{array} & \begin{array}{l}\text { Déterminer le nombre de fréquences des sous-concepts dans chaque } \\ \text { curriculum. Calculer les proportions par rapport au nombre total de } \\ \text { mentions. Établir certaines hypothèses préliminaires quant au niveau } \\ \text { d'intégration de l'approche actionnelle et du plurilinguisme du CECR au } \\ \text { sein de chacun d'entre eux. }\end{array} \\ & \begin{array}{l}\text { Interpréter les tendances conceptuelles des curriculums selon une } \\ \text { hiérarchie d'intégration du CECR et émettre des constats à cet effet. } \\ \text { Effectuer des analyses transversales entre sous-concepts si cela est } \\ \text { pertinent à la question de recherche. }\end{array} \\ \text { Traitement des données } & \end{array}$

Ce modèle nous a permis d'analyser le contenu de ces curriculums de manière exhaustive, puisqu'il permet d'en saisir toute leur complexité conceptuelle. Passons maintenant à la présentation des différentes étapes de l'analyse de contenu que sont l'extraction, l'examen et le traitement des données.

\section{Étape 1 : l'extraction des données}

Les curriculums ont tous été consultés en ligne. À partir de la grille d'extraction (voir Annexe A), il a donc fallu extraire et comptabiliser les données explicites relatives aux sous-concepts présents dans chacun de leur texte. À la lecture des curriculums, nous avons ainsi pu détecter la présence ou l'absence de mentions explicites / unités de sens, soit des synonymes sémantiques, de chaque sous-concept inscrit dans le texte (L'Écuyer, 1987). La comptabilisation du nombre d'occurrences des divers sous-concepts nous a permis d'établir le niveau de leur intégration respective dans le texte (Grbich, 2007). Les unités de sens ne sont pas des répliques exactes des sous-concepts recherchés, car il s'agit de mentions explicites en lien non pas avec leur appellation stricte, mais bien leur sens. À l'aide du logiciel NVivo, les unités de sens repérées ont été classifiées au fil de la lecture dans la grille intégrée à même le logiciel (Grbich, 2007; L'Écuyer, 1987; Van der Maren, 2004). En dernier lieu, les pourcentages ont été calculés pour chaque sous-concept par rapport au nombre total de sous-concepts au sein de chaque curriculum. Cette étape quantitative a ainsi permis la réalisation de l'examen des données.

\section{Étape 2 : l'examen des données}

Pour exécuter l'examen des données, nous avons dans un premier temps rédigé pour chaque curriculum une description basée sur l'ordre de mentions des unités de sens répertoriées et des pourcentages des sous-concepts de l'approche actionnelle et du plurilinguisme (Van der Maren, 2004). Il s'agissait donc, selon la procédure de l'Écuyer 
(1987), de dresser un bilan nous permettant de répondre à notre question de recherche. Nous avons déjà clairement pu constater à cette étape si certains sous-concepts étaient davantage répétés que d'autres, ce qui a rendu possible l'observation préliminaire de certaines tendances, lesquelles ont par la suite été comparées (Berg et Lune, 2012). À cette étape, plus les sous-concepts sont nombreux, plus ils sont intégrés dans le corps sémantique du curriculum. L'ensemble de sous-concepts détectés dans un curriculum équivaut à $100 \%$. Comme mentionné, les pourcentages pour chaque sous-concept sont calculés par rapport au nombre total repéré. Pour comprendre les niveaux d'intégration de chaque sous-concept, nous avons décidé d'établir des intervalles d'intégration calculés en pourcentage. Ceux-ci ont été catégorisés en ordre décroissant : prédominant $(15,0$ à 20,9 $\%)$; majeur (10,0 à 14,9\%); important (5,0 à 9,9\%); moyen (3,0 à 4,9\%); mineur (1,0 à $2,9 \%)$; et absent ( $0 \%$ à $0,9 \%)$. L'ordre décroissant de présentation a été choisi ici afin de mieux représenter la hiérarchie d'intégration, dans la mesure où la prévalence conceptuelle était plus clairement établie de cette façon. Les intervalles fermés ont aussi été choisis, car ils correspondent beaucoup mieux à l'objectif et à la démarche de cette recherche (Riffe et coll., 2019). Ici, les intervalles de proportions se basent sur le contexte généré à partir des données recueillies au sein des curriculums, ce qui a pour effet de cristalliser certaines tendances conceptuelles. Cette étape a ainsi rendu possible la description de ces tendances, qui a par la suite servi à la production des différents bilans (Riffe et coll., 2019). À cette étape, nous avons donc formulé des commentaires sur la valeur numérique de chaque sousconcept relevant de l'approche actionnelle et du plurilinguisme. Cela étant établi, poursuivons avec la troisième étape, soit celle du traitement des données.

\section{Étape 3 : le traitement des données}

Nous avons, à cette étape-ci, traité les tendances observées, avec pour objectif de les convertir en résultats d'analyse (L'Écuyer, 1987; Van der Maren, 2004). Ainsi, à l'aide du logiciel NVivo, nous avons présenté les résultats sous la forme d'histogrammes démontrant le degré d'introduction des divers sous-concepts de l'approche actionnelle et du plurilinguisme dans les curriculums provinciaux et territoriaux de français de base au Canada, de la $9^{\mathrm{e}}$ à la $12^{\mathrm{e}}$ année. Cette étape nous a fourni un portrait global permettant d'adopter une perspective d'ensemble. Ainsi, nous avons pu constater quels sous-concepts prévalaient dans les curriculums. Une hiérarchisation de leur niveau d'intégration a également été possible, ce qui nous a ensuite permis d'établir des tendances positives et négatives quant à l'intégration de chaque sous-concept.

Les trois étapes de cette méthodologie nous ont permis d'opérationnaliser l'ensemble de cette recherche, de l'extraction des données dans les curriculums, en passant par leur examen et leur traitement, le tout à la lumière de la question de recherche ayant guidé l'ensemble de cette thèse. Nous avons ensuite dressé des bilans conceptuels pancanadiens pour l'approche actionnelle et pour le plurilinguisme. Ces bilans ont rendu possible l'interprétation de diverses tendances, nous permettant ainsi d'établir des comparaisons analytiques avec les recherches récentes au Canada. C'est donc de cette partie dont il sera question dans la présentation des résultats. 


\section{Présentation des résultats}

Les résultats seront présentés par province, de la Saskatchewan (1998) à la Colombie-Britannique (2018), soit selon l'ordre chronologique de publication de leur curriculum de français de base. Nous présenterons, en premier lieu, l'ensemble des intervalles d'intégration de tous les curriculums de l'ensemble des provinces au sein d'un même graphique. Par la suite, nous ferons état des intervalles d'intégration de l'approche actionnelle et du plurilinguisme.

\section{Intervalles d'intégration du corpus complet}

Ainsi, la Figure 2 indique, pour chaque curriculum provincial, le pourcentage de sous-concepts par intervalle d'intégration. À gauche, un acronyme est utilisé pour chaque province. Chaque curriculum provincial est classé selon un ordre chronologique de publication. Les couleurs font quant à elles référence aux intervalles d'intégration, lesquels, rappelons-le, nous fournissent une valeur qualitative illustrant de manière plus concrète le niveau d'intégration de chaque sous-concept dans un curriculum donné. Ainsi, dans cette figure, les six niveaux d'intégration sont représentés pour chaque curriculum, et nous donnent un aperçu visuel de la présence de chaque intervalle d'intégration dans chaque curriculum, allant d'une absence d'intégration à un degré d'intégration prédominant.

\section{Figure 2}

Pourcentage des intervalles d'intégration pour chaque curriculum provincial (approche actionnelle et plurilinguisme combinés)

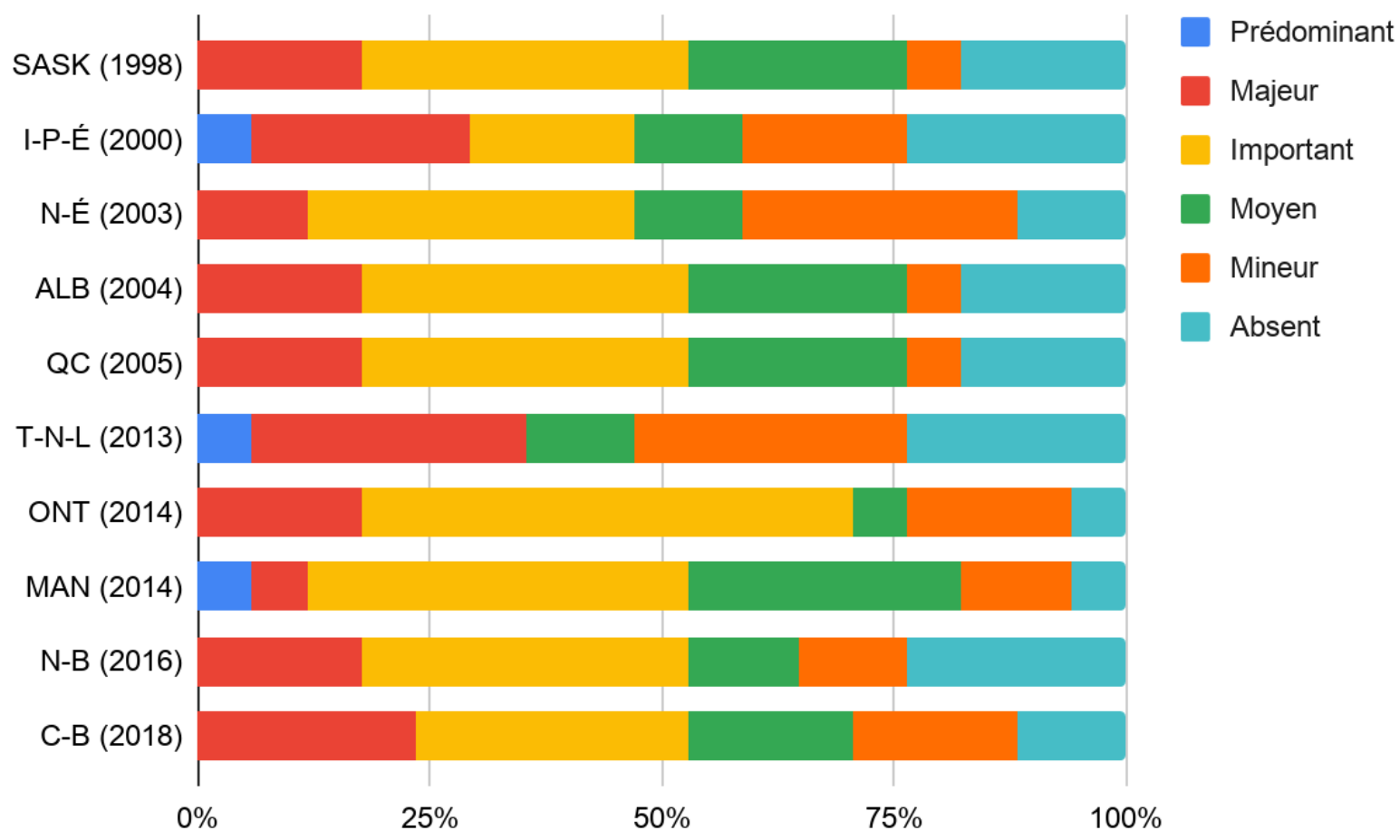


À la lumière de la Figure 2, nous constatons que, dans une perspective pancanadienne, les intervalles d'intégration varient fortement d'un curriculum à l'autre. Certains curriculums semblent plus équilibrés dans la manière dont ils intègrent sémantiquement les sous-concepts, tandis que d'autres mettent bien davantage l'accent sur certains sous-concepts, et ce, au détriment d'autres. Chaque curriculum intègre donc les sous-concepts du CECR à sa façon. Si tous les curriculums avaient intégré les sousconcepts de manière équilibrée, chacune de leur intégration oscillerait autour de $6 \%$. Or, il est possible de constater que certains sous-concepts sont beaucoup plus fortement représentés que d'autres. Nous pouvons donc avancer que chaque curriculum possède une architecture conceptuelle unique, constituée en fonction des besoins pédagogiques propres à sa province. Globalement, nous sommes aussi à même d'observer que tous les intervalles d'intégration sont représentés dans l'ensemble des curriculums. Ainsi, nous pouvons avancer que l'ensemble des curriculums de français de base canadiens du palier secondaire intègrent au moins quelques dimensions conceptuelles du CECR, que ce soit de l'approche actionnelle ou du plurilinguisme. Présentons maintenant les pourcentages relatifs d'intégration pour l'approche actionnelle et pour le plurilinguisme dans tous les curriculums de français de base du palier secondaire au Canada.

\section{Figure 3}

Pourcentage d'intégration de l'approche actionnelle et du plurilinguisme dans les curriculums de français de base du palier secondaire au Canada

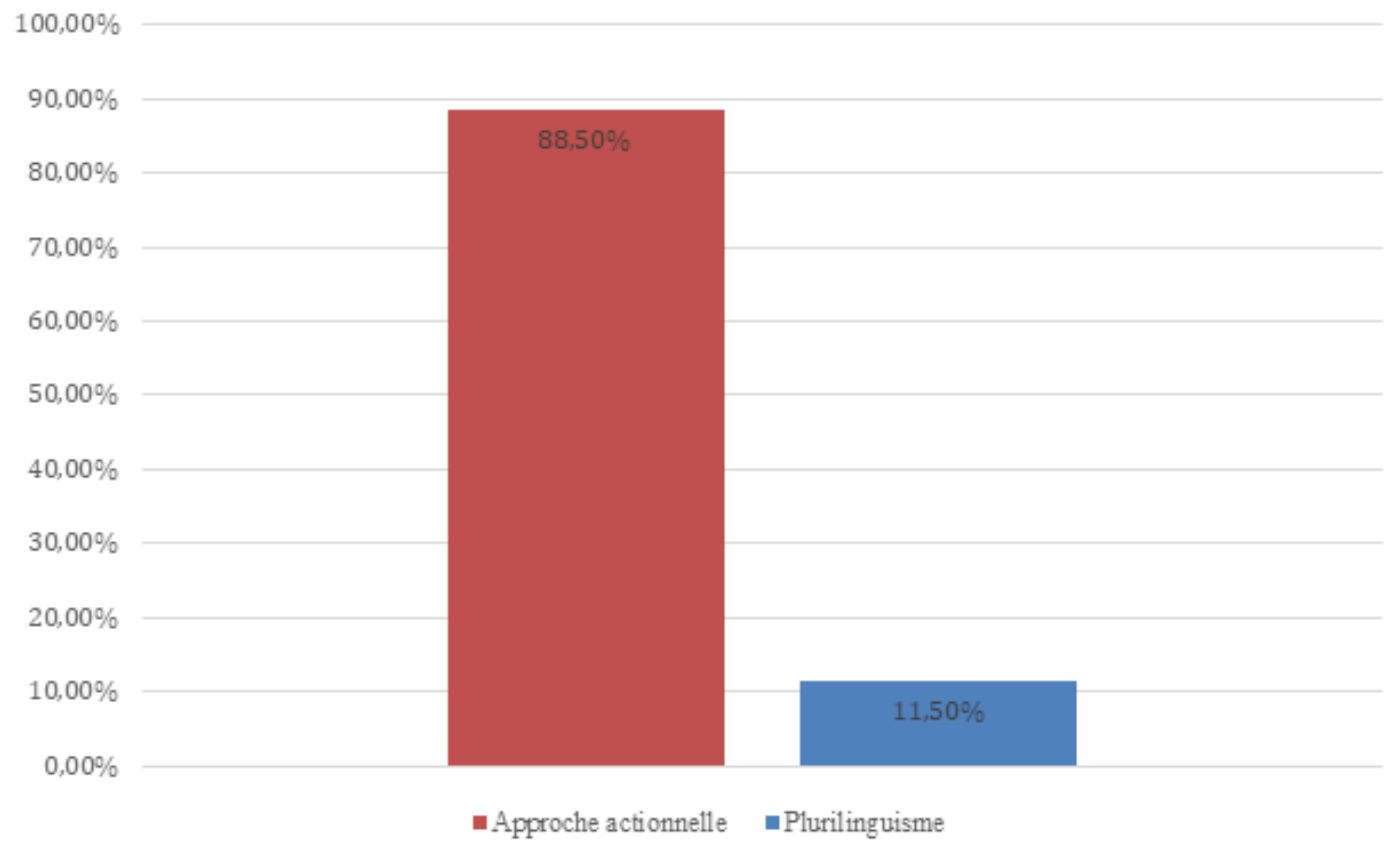

Il est on ne peut plus clair, dans la Figure 3, que l'approche actionnelle domine largement dans les curriculums à l'échelle du Canada, avec une intégration conceptuelle totale de $88,5 \%$. Au contraire, le plurilinguisme n'est présent qu'à 11,5\%. Cela nous permet donc d'avancer que les curriculums de français de base au Canada s'inspirent conceptuellement bien davantage des sous-concepts relatifs à l'approche actionnelle que de 
ceux du plurilinguisme. Par conséquent, si le plurilinguisme est dans son ensemble moins intégré, nous pouvons donc mettre de l'avant, comme nous le verrons ultérieurement, que les dimensions linguistiques et culturelles propres à chaque élève sont beaucoup moins prises en considération dans l'apprentissage du français de base au Canada. Poursuivons la présentation des résultats en lien avec l'intégration conceptuelle de l'approche actionnelle, afin de pouvoir comprendre plus précisément lesquels de ses sous-concepts sont les plus représentés.

\section{Intervalles d'intégration de l'approche actionnelle}

En ce qui concerne l'approche actionnelle, la Figure 4 montre les sous-concepts ainsi que leur degré d'intégration à travers tous les curriculums analysés. Ainsi, dans un premier temps, nous observons que le degré d'intégration diffère grandement d'un sousconcept à l'autre, que ce soit d'une absence d'intégration (p. ex. : médiation) à une domination presque totale (p. ex. : acteur social).

\section{Figure 4}

Intervalles d'intégration des sous-concepts de l'approche actionnelle

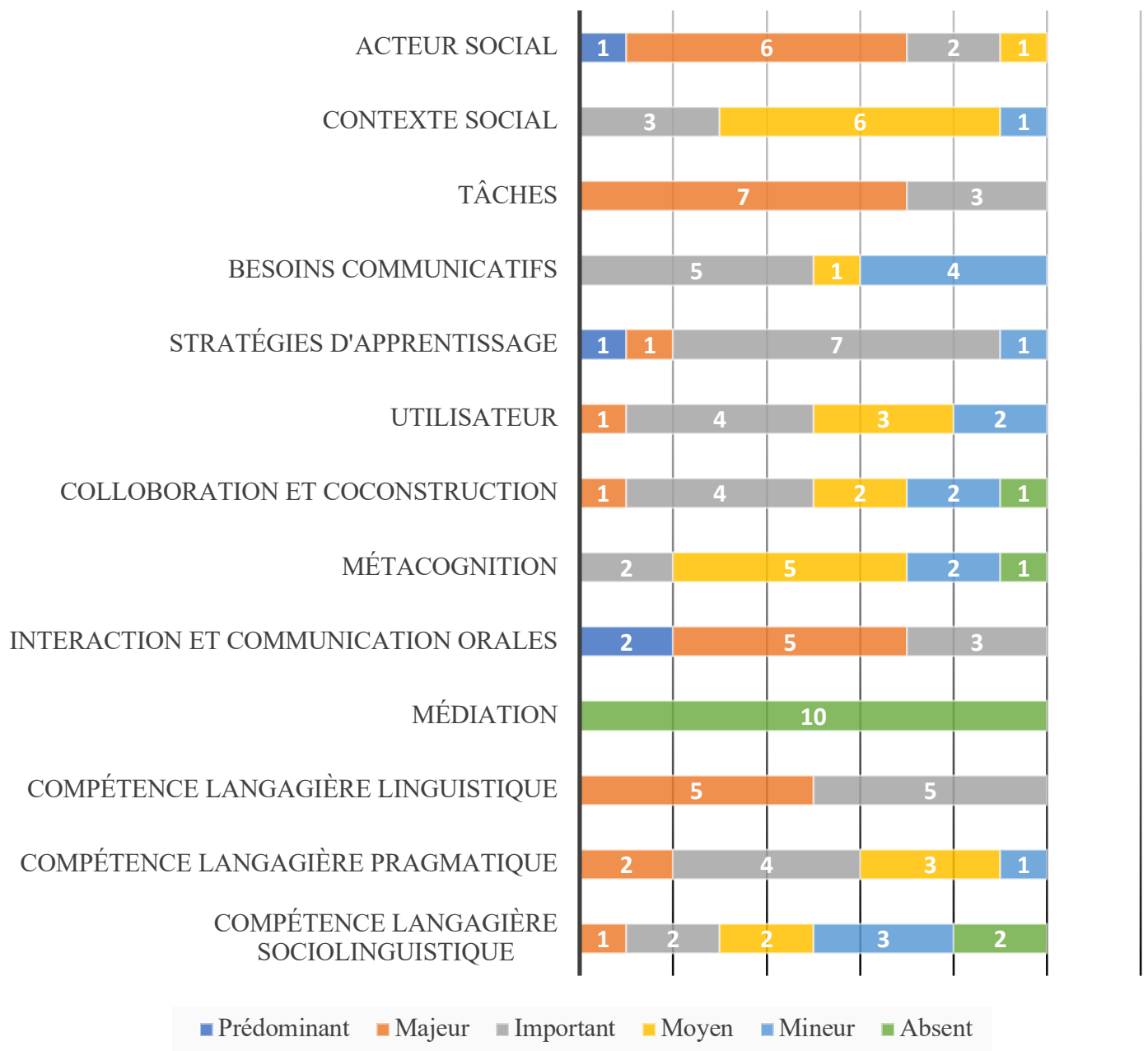


En observant la Figure 3, nous pouvons également souligner quels sous-concepts sont les plus prévalents, à savoir l'acteur social, les tâches, l'interaction et la communication orale ainsi que la compétence langagière linguistique. Ainsi, cela nous renvoie, d'une certaine façon, à un enseignement du français de base mettant de l'avant la réalisation de tâches complexes émulant la vie en société. Pour ce faire, les élèves sont invités à interagir et à communiquer entre eux. Ce portrait global nous montre également que le sous-concept de la collaboration et co-construction fait partie intégrante $\mathrm{du}$ processus d'apprentissage. Ainsi, on encourage fortement les élèves non seulement à travailler en équipe, mais aussi à bâtir les paramètres de leurs projets pour mener à bien, tous ensemble, leurs intentions de communication. Avec la forte présence de l'acteur social, il est également exact d'avancer que, dans l'ensemble, l'élève est perçu avec un certain niveau de complexité sociale. Nous avons également l'impression que la compétence langagière linguistique occupe toujours une place de premier choix dans les priorités pédagogiques, et ce, contrairement aux autres dimensions relatives à l'utilisation de la langue en société (incarnées par les sous-concepts des compétences langagières pragmatique et sociolinguistique). Cela concorde aussi avec la représentation du sousconcept de l'utilisateur, plus ou moins évoqué sur un plan global. Le français revêt ainsi une fonction tout de même plus utilitariste pour l'élève. Le contexte social occupe aussi une place de choix. Cela ne semble toutefois pas toujours être le cas pour les besoins communicatifs de l'élève. Les stratégies d'apprentissage font également partie de l'ensemble des curriculums, et occupent une place somme toute importante dans le texte des curriculums. Cela traduit donc une volonté concrète que les élèves développent davantage leur autonomie d'apprenant. De la même façon, la métacognition se voit représentée dans presque l'ensemble des curriculums, et ce, d'une manière appréciable. Nous pouvons donc en déduire que la plupart des curriculums envisagent le retour réflexif comme une étape importante du processus d'apprentissage du français de base. Enfin, nous constatons dans la Figure 4 l'absence totale du sous-concept de la médiation, ce qui nous permet d'affirmer qu'aucun curriculum ne considère l'élève comme intermédiaire pouvant expliquer et clarifier le sens accordé à une situation donnée à d'autres acteurs sociaux. Maintenant que nous avons globalement présenté l'intégration des sous-concepts de l'approche actionnelle sur le plan pancanadien, il convient de passer aux sous-concepts du plurilinguisme.

\section{Intervalles d'intégration du plurilinguisme}

Rappelons d'emblée que le plurilinguisme, dont les sous-concepts sont le répertoire pluriculturel, le répertoire plurilingue, la compréhension plurilingue et le médiateur interculturel, ne représente que $11,5 \%$ de toutes les unités de sens repérées dans l'ensemble des curriculums de français de base au Canada. Ainsi, le bagage culturel et linguistique composant l'identité de chaque élève ne semble pas faire partie des priorités éducatives dans les curriculums canadiens de français de base, de la $9^{\mathrm{e}}$ à la $12^{\mathrm{e}}$ année. Comme nous pouvons l'apercevoir dans la Figure 5, certains sous-concepts du plurilinguisme semblent être intégrés d'une façon plus importante que d'autres. 


\section{Figure 5}

Intervalles d'intégration des sous-concepts du plurilinguisme

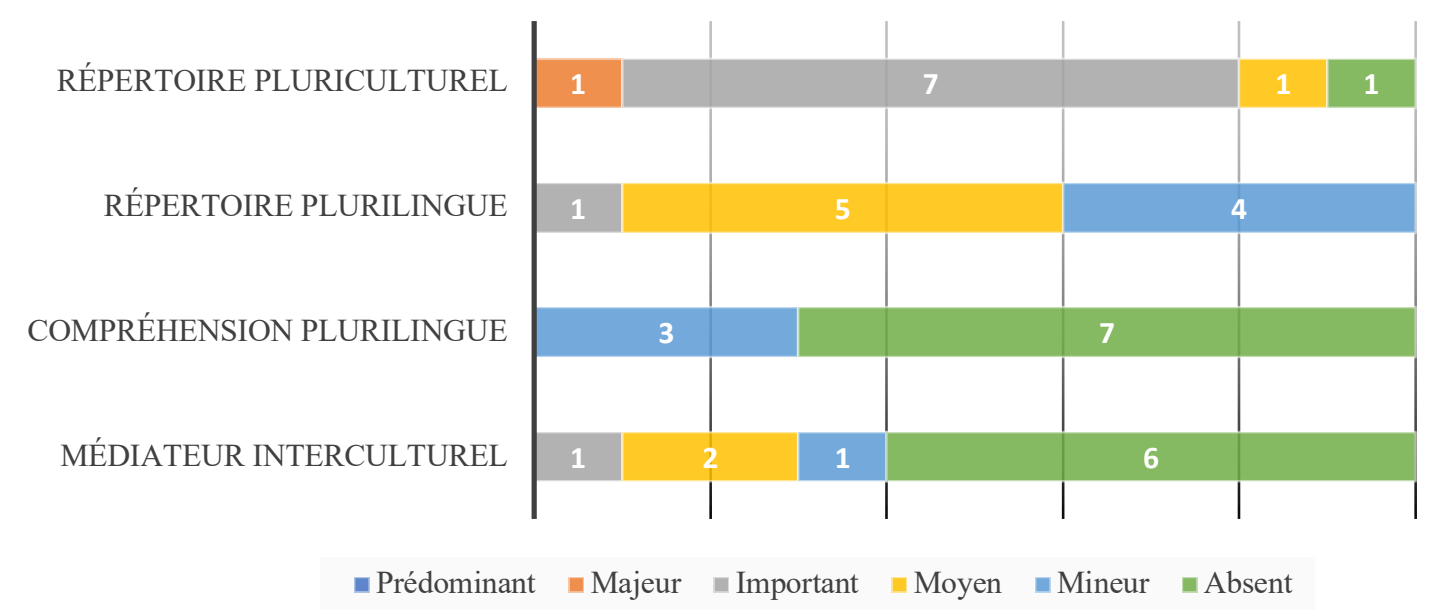

Ainsi, nous observons dans la Figure 5, qu'à l'exception du médiateur interculturel, l'autre sous-concept en lien avec la culture de l'élève (le répertoire pluriculturel) est davantage pris en compte. En revanche, les sous-concepts relatifs à la dimension linguistique de la personne (compréhension plurilingue et répertoire plurilingue) sont très faiblement intégrés, voire carrément absents. Nous pouvons donc avancer que le profil linguistique des élèves, pourtant de plus en plus composite, est faiblement pris en compte dans le corps sémantique des curriculums. De même, la possibilité pour les élèves d'effectuer des allers-retours réflexifs entre leur L1 et leur L2 (compréhension plurilingue) n'y figure tout simplement pas. Cela contraste avec le caractère plus cosmopolite d'une grande partie des classes canadiennes de français de base sur les plans descultures et des langues. Enfin, ce portrait global nous montre que la capacité d'agir des élèves comme intermédiaires de sens dans des situations interculturelles complexes n'est en pratique pas considérée.

Dans cette partie, nous avons donc mis en lumière certains constats découlant de l'interprétation par sous-concept des tendances conceptuelles dégagées à partir de la comptabilisation des unités de sens au sein de chaque curriculum. Cela a rendu possible l'élaboration d'une perspective pancanadienne quant à l'intégration conceptuelle des sousconcepts composant l'approche actionnelle et le plurilinguisme, et, par extension, du CECR.

\section{Discussion}

Dans cette partie, nous comparerons les interprétations mentionnées précédemment avec l'état de la recherche actuelle relative au CECR au Canada. Avant toute chose, attardons-nous par ailleurs au fait que certains curriculums n'ont pas été mis à jour depuis plus d'une décennie. Réitérons de plus qu'un curriculum incarne les priorités pédagogiques du gouvernement en place. À cet égard, quatre curriculums n'ont pas été modifiés depuis plus de quinze ans. Ainsi, cela peut donner l'impression d'un manque flagrant de volonté politique pour réformer ces documents, ce qui contraste avec le fait que les appels au changement sont survenus depuis le début du 21e siècle. Tout de même, malgré cet écart 
de publication entre certains curriculums et le CECR, il nous a été possible d'observer que tous les curriculums de français de base canadiens sont variablement influencés par certains sous-concepts de l'un et/ou de l'autre des concepts piliers du CECR, soit l'approche actionnelle et le plurilinguisme. Cette influence conceptuelle varie certes grandement d'un curriculum à l'autre, mais il n'en demeure pas moins qu'une partie de leur architecture conceptuelle est inspirée du CECR, tout en reconnaissant que l'influence d'un tel outil prend du temps avant de se manifester dans le corps des documents. À ce titre, à l'échelle canadienne, nous pouvons affirmer que l'approche actionnelle est plus prévalente que le plurilinguisme. De plus, comme l'avait suggéré Vandergrift (2006) toutes les compétences langagières sont traitées dans les curriculums, mais de manière toutefois inégale. Pour lui, la métacognition devait également jouer un rôle important dans le processus d'apprentissage du FLS. C'est ce que nous remarquons effectivement dans bon nombre de curriculums, quoique à géométrie variable. Ainsi, les élèves sont encouragés, dans l'ensemble, à évaluer leurs propres apprentissages. Vandergrift soulignait par ailleurs l'importance primordiale de considérer la langue cible comme un véritable outil de communication. À ce titre, nous avons observé une intégration forte des sous-concepts d'utilisateur, de tâches et de contexte social, ainsi que de l'interaction et communication orales. Piccardo (2016b) faisait aussi mention de l'importance d'utiliser la langue cible pour bâtir des apprentissages réels par le biais d'une interaction sociale et authentique. Ainsi, nous remarquons qu'un bon nombre de sous-concepts de l'approche actionnelle sont fortement pris en compte, alors que d'autres ne le sont pas.

L'approche actionnelle repose entre autres sur une répartition équilibrée des trois compétences langagières (linguistique, pragmatique et sociolinguistique). Une tâche n'intégrant pas ces trois compétences ne peut pas être accomplie dans toute sa complexité (Conseil de l'Europe, 2001). En effet, communiquer une intention de communication requiert leur mise en œuvre complète (Rosen, 2010). Dans le portrait pancanadien de l'intégration, nous avons constaté que la compétence linguistique revêt davantage d'importance que les deux autres. Ainsi, l'apprentissage du code linguistique semble toujours figurer comme un élément central de l'apprentissage du français de base au Canada. Nous ne pouvons donc affirmer que toutes les dimensions de l'approche actionnelle soient intégrées de manière égale. À ce titre, la simulation de véritables tâches complexes et authentiques s'en trouve donc amenuisée. Cela rend ainsi plus ardue la tâche d'élargir les perspectives d'apprentissage de la L2 au-delà des murs de la classe (Piccardo, 2016b).

Par ailleurs, Piccardo (2016a) avance que l'interaction sociale entre pairs revêt une importance capitale pour établir la rétroaction nécessaire à tout apprentissage linguistique, car c'est notamment par l'échange que l'élève se corrige et s'améliore. La forte majorité des curriculums de français de base au Canada promeut un apprentissage du français de base axé sur l'interaction et la communication orales. En outre, une si grande intégration de ce sous-concept pourrait potentiellement expliquer pourquoi la collaboration et la coconstruction sont plus faiblement incorporées. Ainsi, les élèves de français de base au Canada développent des apprentissages linguistiques au sein d'un contexte social qu'ils contribuent à définir.

En ce qui concerne la métacognition, Little et Taylor (2013) mettent aussi de l'avant qu'il s'agit d'une dimension cruciale dans l'apprentissage d'une L2. Ainsi, en effectuant des retours réflexifs, les élèves déterminent si les résultats de la tâche 
concordent avec les objectifs. La Figure 2, vue précédemment, portant sur les pourcentages des intervalles d'intégration pour chaque curriculum provincial (approche actionnelle et plurilinguisme combinés), nous montre que l'intégration de ce sous-concept n'est pas répartie de manière égale dans l'ensemble du corps sémantique étudié. En effet, certains curriculums le considèrent comme une pierre d'assise, alors que d'autres ne font qu'à peine l'effleurer.

Little et Taylor (2013) soulignaient également qu'une classe de FLS inspirée de l'approche actionnelle faisait en sorte que les élèves étaient davantage motivés, et que leur autonomie en devenait rehaussée. Ainsi, ils ont remarqué que les élèves faisaient davantage usage du français lorsqu'ils réalisaient des projets authentiques simulant la vie réelle. Notre perspective pancanadienne nous montre que cet aspect du CECR revêt une importance de premier plan. À ce sujet, nous avons vu qu'un accent particulier était accordé aux tâches et à l'acteur social. Les élèves sont donc en mesure de comprendre clairement les tenants et aboutissants de l'utilisation de la langue cible, car elle devient un outil de communication efficace dans leur propre vie (Piccardo, 2013a). Par ailleurs, nous avons observé que le sous-concept de la médiation est absent sur le plan sémantique dans l'ensemble des curriculums. Or, cela n'a rien d'étonnant, puisqu'il a été introduit dans le CECR en 2018, soit lors de l'année de publication du dernier curriculum de français de base au Canada. Cela dit, le CECR ne se compose pas que de l'approche actionnelle, le plurilinguisme incarnant en effet lui aussi un pilier sur lequel cet outil est fondé.

À ce titre, Vandergrift (2006) avait déjà mentionné que la démographie des élèves canadiens était en constante transformation, vu l'augmentation sans cesse accrue de l'immigration internationale. Ainsi, les profils culturels et linguistiques des apprenants sont beaucoup plus diversifiés qu'au siècle précédent. Il est donc crucial de tenir compte de cette nouvelle réalité sociolinguistique pour réaliser des apprentissages dans la L2, tel que le soutenait Vandergrift. Moore et Castellotti (2008) affirment elles aussi que les sociétés contemporaines sont plus complexes sur ce plan, et qu'il est désormais impératif de tenir compte de cette réalité culturelle et linguistique composite. Pourtant, notre perspective pancanadienne nous montre clairement que cet aspect n'est presque aucunement reflété dans l'ensemble des curriculums canadiens de français de base du palier secondaire.

Rappelons en effet que le plurilinguisme ne recueille que $11,5 \%$ des unités de sens totales. Cela n'a rien de surprenant, dans la mesure où ce concept a émergé de manière plus concrète en 2009 à la suite des recherches de Coste, Moore et Zarate (2009) pour le compte du CoE. On semble ainsi percevoir davantage l'élève dans ses dimensions individuelle et sociale, plutôt que linguistique et culturelle.

Piccardo (2016a) a été on ne peut plus claire à ce sujet. Le plurilinguisme incarne un pilier fondamental du CECR, et il doit de ce fait même être pris en compte. L'élève doit pouvoir s'appuyer sur ses repères linguistiques et culturels pour réaliser des apprentissages dans sa L2. Cela dit, le portrait de l'intégration globale du plurilinguisme doit être quelque peu nuancé. En effet, si les sous-concepts relatifs à la dimension linguistique du plurilinguisme sont pratiquement absents, nous avons observé que les curriculums de français de base canadiens accordent toutefois une certaine place à la culture des élèves dans le processus d'apprentissage, avec une intégration appréciable du répertoire pluriculturel. Moore et Castellotti (2008) soulignaient par ailleurs qu'une pédagogie de la L2 axée sur les particularismes linguistiques et culturels contribue à individualiser, et ainsi à améliorer, le processus d'apprentissage. Nous avons ici observé que les curriculums de 
français de base canadiens ne permettent pas, dans l'ensemble, une individualisation à cet égard. En revanche, nous avons remarqué que les élèves sont davantage perçus comme des acteurs sociaux agissant au sein de contextes sociaux. Leur identité plurielle n'est donc pas totalement prise en compte, et ce, malgré la mondialisation complexifiant cette même notion (Moore et Castellotti, 2008). À ce titre, la quasi-absence sémantique du médiateur interculturel ne peut être que plus probante. Dans des contextes sociaux plus complexes, les élèves devraient être en mesure d'utiliser toutes leurs ressources linguistiques et culturelles (Moore et Castellotti, 2008). La capacité de composer avec l'ensemble de cette diversité n'est donc ici pas encore au rendez-vous.

En ce qui concerne la prise en compte de la pluralité linguistique, dans l'ensemble elle n'y est tout simplement pas. L'hétérogénéité linguistique ne fait en effet aucunement partie du processus d'apprentissage de la L2, et ce, dans l'ensemble des curriculums de français de base canadiens. Pourtant, Piccardo (2016a) a clairement souligné les avantages pédagogiques d'élargir les horizons linguistiques de la classe de L2 pour les élèves, soit de ne pas les limiter qu'au seul usage de la langue cible. En effet, les élèves doivent être en mesure d'utiliser toutes leurs ressources linguistiques pour réaliser des apprentissages en L2 (Piccardo, 2016a), étant donné que leur apprentissage n'est pas linéaire, demandant des allers-retours constants avec l'ensemble des composantes linguistiques d'une personne (Piccardo, 2016a). Il est clair toutefois que cela n'est pas le cas ici, comme nous avons pu le constater antérieurement. Il est donc ardu pour les élèves d'utiliser leur bagage linguistique, ce qui peut surprendre compte tenu de la complexification linguistique et culturelle croissante d'un Canada de plus en plus diversifié démographiquement (Statistique Canada, 2017). Finalement, l'état actuel de la recherche montre aussi que les classes de français de base n'ont pas encore intégré suffisamment le plurilinguisme dans l'apprentissage d'une L2, malgré le fait qu'il s'agit d'un élément conceptuel pourtant fondamental du CECR. A contrario, on semble encore promouvoir une dimension exclusive de l'utilisation du français comme L2 dans le processus d'apprentissage (Cummins et Swain, 2014; Piccardo et Capron Puozzo, 2015; Taylor et Culter, 2016; Wernicke et Bournot-Trites, 2011). Ainsi, les répertoires plurilingues et pluriculturels se retrouvent clairement à l'arrière-plan, ce qui concorde également avec nos résultats de recherche. Cela peut surprendre à bien des égards, étant donné qu'il a été montré à quel point les élèves bénéficient de pouvoir s'appuyer sur leur L1 pour réaliser des apprentissages linguistiques dans leur L2 (Cummins et Swain, 2014).

\section{Conclusion}

En définitive, à la lumière du prisme conceptuel du CECR, ce projet de recherche a permis de produire une analyse de la vision de l'apprentissage, de l'enseignement, et de l'évaluation du français de base au Canada à travers une étude approfondie des curriculums de français de base du palier secondaire de toutes les provinces et territoires. Notons qu'il est aussi fort plausible que certains curriculums parus avant le CECR (Conseil de l'Europe, 2001) aient été influencés par cet outil, étant donné que certains sous-concepts le constituant étaient en phase avec les approches pédagogiques du temps. En effet, il est dorénavant possible de conclure que la plupart des curriculums de français de base au Canada s'inspirent, d'une façon ou d'une autre, de sous-concepts constitutifs de l'approche actionnelle ou du plurilinguisme. C'est au niveau de leur introduction respective dans les 
curriculums que la donne change. Ainsi, un premier constat s'impose : l'approche actionnelle est de loin plus présente que le plurilinguisme conceptuellement parlant. Pour l'approche actionnelle, les sous-concepts suivants sont les plus prégnants sémantiquement : l'acteur social, les tâches, l'interaction et la communication orales, la compétence langagière linguistique et les stratégies d'apprentissage. Cela ne suffit toutefois pas, comme en témoigne l'appel lancé par Arnott et coll. (2017) d'introduire plus exhaustivement le CECR, et ce, dans son entièreté conceptuelle. En effet, le plurilinguisme ne représente que $11,5 \%$ des unités de sens recensées. L'incorporation des deux concepts fondamentaux doit donc impérativement continuer afin de pouvoir véritablement observer toute l'étendue du potentiel pédagogique du CECR en classe de FLS (Arnott et coll., 2017).

La principale contribution de ce projet de recherche est donc, en fin de compte, de proposer une vision globale des buts relatifs à l'apprentissage, à l'enseignement et à l'évaluation du français de base des provinces et territoires canadiens. Cette analyse aura permis de confirmer ou d'infirmer si les décideurs des différents ministères de l'Éducation ont intégré les suggestions du CMEC (2010) en lien avec le CECR, et de surcroît à quel degré. Les résultats découlant de ce projet serviront de tremplin à de futures recherches dans le domaine des politiques éducatives ayant pour but de poursuivre l'incorporation du CECR au Canada, comme souhaité par Arnott et coll. (2017). De nouvelles pistes de recherche en lien avec le français de base pourraient également être développées, étant donné que $75 \%$ des élèves canadiens qui fréquentent les écoles de langue anglaise y sont actuellement inscrits (Canadian Parents for French, 2019). Tout d'abord, une revue du degré d'intégration du CECR dans les curriculums d'immersion française pourrait aussi être effectuée, et ce, à dessein d'ensuite la comparer avec la présente étude. Dans la même veine, une analyse comparative pourrait être menée avec les curriculums de français de base du palier élémentaire, laquelle pourrait être ensuite être mise en parallèle avec ce projet de recherche portant sur le palier secondaire. Il conviendrait en outre de s'interroger sur les facteurs sous-jacents à la faible intégration du plurilinguisme dans les curriculums de français de base du palier secondaire au Canada. Une telle analyse serait des plus pertinentes, étant donné que le Canada a constitutionnalisé le multiculturalisme. En outre, soulignons que le développement d'un guide précis à la mise en œuvre du CECR au Canada serait des plus bénéfiques, dans la mesure où il assurerait la liaison entre les spécificités canadiennes et le CECR. Dans une perspective plus internationale, une comparaison des curriculums canadiens avec ceux d'autres fédérations bilingues ou multilingues pourrait être entreprise, et ce, pour dégager certaines convergences et divergences dans leur intégration respective du CECR. En somme, le présent projet constituera une nouvelle brique dans ce grand édifice qu'est la recherche relative au FLS au Canada, pays qui, rappelons-le, a énoncé à de maintes reprises déjà sa volonté d'accroitre le bilinguisme des élèves canadiens (CMEC, 2010), et ce, ad mare usque ad mare. 


\section{Références}

Arnott, S., Brogden, L. M., Faez, F., Péguret, M., Piccardo, E., Rehner, K., Taylor, S. K. et Wernicke, M. (2017). The Common European Framework of Reference (CEFR) in Canada: A Research Agenda. Revue canadienne de linguistique appliquée, 20 (1), 31-54.

Berg, B. L. et Lune, H. (2012). Qualitative Research Methods for the Social Sciences (8 éd.). New York: Pearson Education Inc.

Canadian Parents for French (2019). French As A Second Language Enrolment Statistics. Récupéré le 10 mars 2020 du site de l'organisme. https://cpf.ca/en/files/FSLEnrolment-Stats-2018-2019.pdf

Comité permanent des langues officielles (2007). Les programmes d'enseignement du français langue seconde au Canada : État des lieux. Chambre des Communes, Canada.

Conseil de l'Europe (2001). Cadre européen commun de référence pour les langues: apprendre, enseigner, évaluer. Récupéré le 20 octobre 2018 du site de l'organisme. https://rm.coe.int/16802fc3a8

Conseil de l'Europe (2018). Cadre européen commun de référence pour les langues : apprendre, enseigner, évaluer. Volume complémentaire avec de nouveaux descripteurs. Récupéré le 20 octobre 2018 du site de l'organisme. https://rm.coe.int/cecr-volume-complementaire-avec-de-nouveauxdescripteurs/16807875d5

Conseil de l'Europe (2020). Common European Framework of Reference for Languages: Learning, teaching, assessment - Companion volume, Council of Europe Publishing, Strasbourg. Récupéré le 8 septembre du site web de l'organisme. www.coe.int/langcefr

Conseil des ministres de l'Éducation (Canada) / CMEC. (2010). L'exploitation du Cadre européen commun de référence pour les langues (CECR) dans le contexte canadien. Récupéré le 20 octobre 2018 du site de l'organisme. http://cmec.ca/docs/CECRcontexte-canadien.pdf

Conseil des ministres de l'Éducation (Canada) / CMEC. (2015). Le français langue seconde au Canada : potentiel de collaboration. Récupéré le 20 octobre 2018 du site de l'organisme.

https://www.cmec.ca/Publications/Lists/Publications/Attachments/370/ACPLSRapport-FLS-au-Canada-FR.pdf

Coste, D., Moore, D. et Zarate, G. (2009). Plurilingual \& pluricultural competence. Strasbourg, France: Council of Europe (Language Policy Division).

Creswell, J. W. (2019). Educational Research: Planning, Conducting and Evaluating Quantitative and Qualitative Research. 6e édition. Boston, États-Unis: Pearson

Cummins, J. et Swain, M. (2014). Bilingualism in education: aspects of theory, research and practice. Routledge.

Dagenais, D. (2008). La prise en compte du plurilinguisme d'enfants issus de familles immigrantes en contexte scolaire : une analyse de cas. Revue des sciences de l'éducation, 34(2), 351-375. 
Faez, F., Majhanovich, S., Taylor, S. K., Smith, M. et Crowley, K. (2011). The power of "Can Do" statements: Teachers' perceptions of CEFR-informed instruction in French as a second language classrooms in Ontario. Revue canadienne de linguistique appliquée, 14 (2), 1-19.

Germain, C. (2013). Évolution de l'enseignement des langues: 5000 ans d'histoire. Paris: CLE international.

Grbich, C. (2007). Qualitative Data Analysis: An Introduction. London: SAGE Publications Ltd.

Karsenti, T. et Savoie-Zajc, L. (2011). La recherche en éducation : étapes et approches (Troisième édition.). Saint-Laurent: ERPI.

Lapkin, S., Mady, C. et Arnott, S. (2009). Research perspectives on core French: A literature review. Revue canadienne de la linguistique appliquée, 12 (2), 6-30.

L'Écuyer, R. (1987). L'analyse de contenu : notion et étapes. Deslauriers, J-P. (dir.), Les méthodes de la recherche qualitative (p. 49-65). Québec, Canada : Presses de l'Université du Québec.

Le Thiec Rautureau, M. (2011). Un cadre de référence pour le Canada. Québec français, $163,50-51$.

Little, D. et Taylor, S. K. (2013). Tirer des leçons des recherches empiriques sur la mise en œuvre du Cadre européen commun de référence pour les langues et du Portfolio européen des langues pour les recherches futures. Édition spéciale de la Revue canadienne des langues modernes, 69(4), 1-23.

Martyniuk, W. et Noijons, J. (2007). Synthèse des résultats d'une enquête sur l'utilisation $d u$ CECR au niveau national dans les États membres du Conseil de l'Europe. Communication présentée au forum politique : Le Cadre européen commun de référence pour les langues (CECR) et l'élaboration de politiques linguistiques : défis et responsabilités, Strasbourg, France. Récupéré le 2 octobre 2018 du site de l'Association des professeurs de langues vivantes (APLV) : https://www.aplvlanguesmodernes.org/spip.php?article774

Ministère de l'Éducation de l'Alberta (2004). CECRL French as a second language nineyear program of studies (grade 4 to grade 12). Récupéré le 5 février 2019 du site de l'organisme: https://education.alberta.ca/media/160306/nine year.pdf

Ministère de l'Éducation de la Colombie-Britannique (2018). Core French. Récupéré le 5 février 2019 du site de l'organisme : https://curriculum.gov.bc.ca/curriculum/secondlanguages/all/courses

Ministère de l'Éducation de l'Île-du-Prince-Édouard (2000). Français de base :

programme d'études et guide pédagogique, de la $10^{e}$ à la $12^{e}$ année. Récupéré le 5 février 2019 du site de l'organisme :

https://www.princeedwardisland.ca/en/information/education-and-lifelonglearning/core-french-curriculum

Ministère de l'Éducation du Manitoba (2014). French Communication and Cultures: grades 4 to 12. Récupéré le 5 février 2019 du site de l'organisme : https://www.edu.gov.mb.ca/k12/cur/french/comm_culture.html

Ministère de l'Éducation du Nouveau-Brunswick (2016). Post-Intensive French - Grades 9-12. Récupéré le 5 février 2019 du site de l'organisme: https://www2.gnb.ca/content/gnb/en/departments/education/k12/content/anglophone _sector/curriculum_anglophone.html\#3 
Ministère de l'Éducation de la Nouvelle-Écosse (2003). Français de base-secondaire $2 e$ cycle

10e à la 12e année. Récupéré le 5 février 2019 du site de l'organisme:

https:/curriculum.novascotia.ca/core-french/course/francais-de-base-10e-a-12eannee-core-french-10-12

Ministère de l'Éducation de l'Ontario (2014). French as a Second Language. Récupéré le 5 février 2019 du site de l'organisme:

http://www.edu.gov.on.ca/eng/curriculum/secondary/fsl.html

Ministère de 1'Éducation du Québec (2005). Français langue seconde (programme de base). Récupéré le 5 février 2019 du site de l'organisme:

http://www.education.gouv.qc.ca/en/teachers/quebec-education program/secondary/languages/french-as-a-second-language/

Ministère de l'Éducation de la Saskatchewan (1998). Core French A Curriculum Guide for the Secondary Level. Récupéré le 5 février 2019 du site de l'organisme:

https://curriculum.gov.sk.ca/webapps/moe-curriculum-

BBLEARN/CurriculumHome?id=404\&language $=\mathrm{fr}$

Ministère de l'Éducation de Terre-Neuve-et-Labrador (2013). Senior High Core French. Récupéré le 5 février 2019 du site de l'organisme: https://www.gov.nl.ca/eecd/k12/curriculum/guides/corefrench/

Moore, D. et Castellotti, V. (2008). Conférence internationale sur l'acquisition d'une $3 e$ langue et le plurilinguisme. (n.d.). La compétence plurilingue: Regards francophones (Transversales (Berne, Suisse) ; v. 23). Berne; New York, NY: Lang.

North, B. (2000). The development of a common framework scale of language proficiency. New York: Peter Lang.

Piccardo, E. (2013a). (Re) conceptualiser l'enseignement d'une langue seconde à l'aide d'outils d'évaluations: comment les enseignants canadiens perçoivent le CECR. La Revue canadienne des langues vivantes, 69 (4), 386-414.

Piccardo, E. (2013b). Le CECR au Canada : entre réalité et représentation. In V. Bigot, A. Bretegnier et M. Vasseur (Réd.), Plurilinguisme ? 20 ans après. (pp. 65-73). Paris: Edition des archives contemporaines.

Piccardo, E. et Capron Puozzo, I. (2015). From second language pedagogy to the pedagogy of "plurilingualism": A possible paradigm shift? La Revue canadienne des langues vivantes, 71 (4), 317-499.

Piccardo, E. (2016a). La diversité culturelle et linguistique comme ressource à la créativité. Voix plurielles, 13 (1), 57-75.

Piccardo, E. (2016b). Un parcours de recherche-développement vers 1'approche actionnelle : un projet canadien. Les Langues modernes, La perspective actionnelle : état des lieux 3, 31-41.

Piccardo, E. (2017). Plurilingualism as a Catalyst for Creativity in Superdiverse Societies: A Systemic Analysis. Frontiers in Psychology, 8, 2169-2169. https://doi.org/10.3389/fpsyg.2017.02169

Piccardo, E. et North, B. (2019). The Action-Oriented Approach: A Dynamic Vision of Language Education. Bristol: Multilingual Matters. 
Piccardo, E., North, B. et Maldina, E. (2019). Promoting innovation and reform in language education through a Quality Assurance template for CEFR implementation. Canadian Journal of Applied Linguistics / Revue canadienne de linguistique appliquée, Édition spéciale 22(1), 103-128.

Reis, G. et Ng-A-Fook, N. (2010). TEK talk: so what? Language and the Decolonization of Narrative Gatekeepers of Science Education Curriculum. Cultural Studies of Science Education, 5, 1009-1026.

Riffe, D., Lacy, S., Watson, B. et Fico, F. (2019). Analyzing media messages: using quantitative content analysis in research (Fourth edition.). New York: Routledge.

Rosen, E. (2010). Perspective actionnelle et approche par les tâches en classe de langue. La Revue canadienne des langues vivantes, 66 (4), 487-498.

Statistique Canada (2017). Immigration et diversité ethnoculturelle : faits saillants du Recensement de 2016. Récupéré le 23 mars 2021 du site de l'organisme : https://www150.statcan.gc.ca/n1/daily-quotidien/171025/dq171025b-fra.htm

Taylor, S. K. et Cutler, C. (2016). Showcasing the translingual SL/FL classroom: Strategies, practices, and beliefs. La Revue canadienne des langues vivantes, 72 (4), 389-422.

Vandergrift, L. (2006). Nouvelles perspectives canadiennes. Proposition d'un cadre commun de référence pour les langues pour le Canada. Bibliothèque et Archives Canada.

Vandergrift, L. (2015). The DELF in Canada: Perceptions of Students, Teachers, and Parents. La revue canadienne des langues vivantes, 71 (1), 52-74.

Van der Maren, J-M. (2004). Méthodes de recherche pour l'éducation, 2e édition. Bruxelles, Belgique : De Boeck.

Wernicke, M. et Bournot-Trites, M. (2011). Introducing the CEFR in BC: Questions \& challenges. Revue canadienne de linguistique appliquée, 14, 106-128. 


\section{Annexes \\ Annexe A \\ Grille d'extraction : unités de classification / codages}

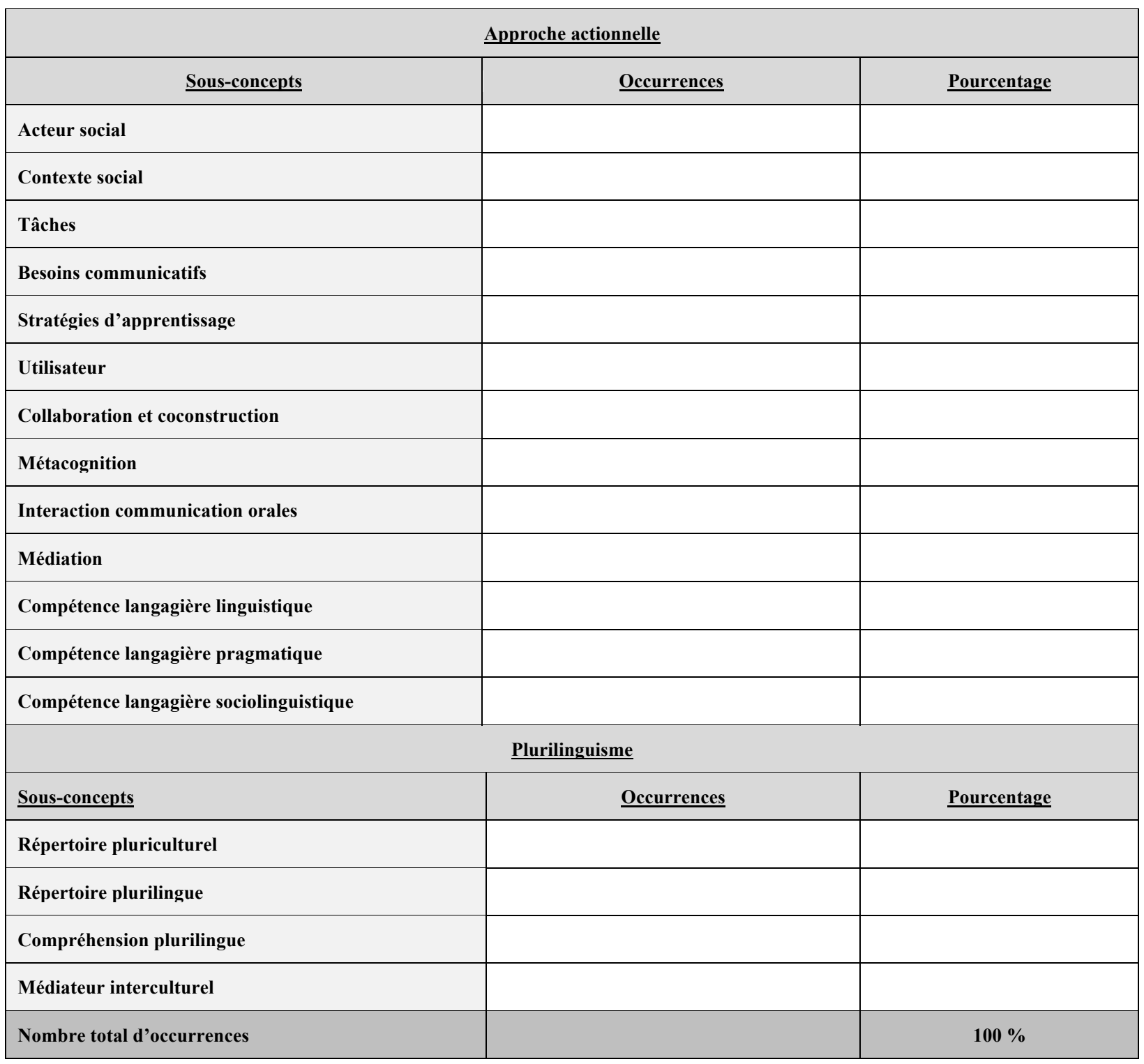




\section{Annexe B \\ Définitions des sous-concepts du Cadre Européen Commun de Référence inspirées des documents du CECR (Le Conseil de l'Europe, 2001, 2018)}

Sous-concepts de l'approche actionnelle

Acteur social

Contexte social

Tâches

\section{Besoins communicatifs}

\section{Stratégies d'apprentissage}

\section{Définitions}

L'apprenant/utilisateur de langues est considéré comme un "acteur social», c'est-à-dire qu'il incarne une personne avec ses caractéristiques personnelles et sociales propres, et agissant au sein de son contexte (social, économique et politique) où il détermine son devenir et exerce un rôle de premier plan dans son processus d'apprentissage de la L2.

Terme anglais équivalent : Social Actor

Le contexte social désigne l'ensemble des dimensions relatives au monde réel dans lequel évolue l'apprenant/utilisateur de langues, qu'elles soient économiques, politiques ou sociales.

Terme anglais équivalent : Social Context

Les tâches sont des projets pédagogiques d'envergure, pour lesquels les apprenants/utilisateurs de langues planifient, recherchent et exécutent des actions sociales de différents niveaux de difficulté reflétant la vie en société.

Terme anglais équivalent : Authentic Task

Les besoins communicatifs constituent ce qui doit être pris en considération dans le processus d'acquisition de la L2 afin que l'apprenant/utilisateur de langues puisse être en mesure de réaliser ses intentions de communication dans le cadre des tâches à effectuer.

Terme anglais équivalent : Communicative Needs

Les stratégies d'apprentissage représentent tout ce que l'apprenant/utilisateur peut utiliser, à toutes les étapes du processus d'apprentissage, pour l'aider à développer son autonomie à dessein d'accomplir les tâches émulant la vie en société.

Terme anglais équivalent : Learning Strategies 


\section{Utilisateur}

\section{Collaboration et coconstruction}

\section{Métacognition}

\section{Interaction et communication orales}

L'apprenant/utilisateur de langues est considéré comme une personne à part entière qui a besoin de la langue pour communiquer ses intentions aux autres acteurs sociaux présents dans son contexte social. La langue est donc ici considérée en tant que moyen de communication et non comme un objet d'étude isolé de son contexte social, économique, culturel et politique.

Terme anglais équivalent : User

La collaboration et coconstruction consiste en une stratégie d'enseignement mettant de l'avant le travail en commun des apprenants/utilisateurs de langues, dans un premier temps pour déterminer les buts d'apprentissage et le sens de la tâche, ainsi que les stratégies d'apprentissage à adopter, et, dans un deuxième temps, pour mener à bien toutes les étapes relatives à sa réalisation.

Terme anglais équivalent: Teamwork And Coplanning

La métacognition constitue l'étape finale du processus d'apprentissage, où l'apprenant/utilisateur de langues effectue un retour réflexif et critique sur l'ensemble des étapes relatives au processus d'apprentissage d'une tâche authentique, et ce, à dessein de développer son autonomie lors de la réalisation de tâches subséquentes pour atteindre un niveau d'utilisation indépendant dans la L2.

Terme anglais équivalent : Self-assessment

L'interaction et communication orales représentent un aspect fondamental de l'approche actionnelle, mettant de l'avant le fait que les apprenants/utilisateurs de langues, considérés comme des acteurs sociaux, doivent communiquer leurs idées par des conversations lors de la réalisation des tâches, notamment avec les acteurs sociaux que sont les autres apprenants/utilisateurs de langues. Le but est ici de simuler la vie réelle où les personnes communiquent essentiellement de manière orale entre elles.

Terme anglais équivalent: Oral interaction And Communication 
Médiation

Compétence langagière linguistique

Compétence langagière pragmatique

Compétence langagière sociolinguistique
La médiation est l'aptitude d'un apprenant/utilisateur de langues à agir comme intermédiaire, en expliquant dans une situation donnée le sens accordé à une situation et d'en clarifier le but à d'autres acteurs, afin que ceux-ci soient en mesure de comprendre, d'agir et/ou de produire selon le sens voulu dans le contexte de la situation donnée.

Terme anglais équivalent : Mediation

La compétence langagière linguistique représente la capacité de l'apprenant/utilisateur de langues à connaître et à comprendre les dimensions objectives et formelles de la langue comme code de communication : le lexique, la grammaire, la sémantique, la phonologie et l'orthographe. Sa connaissance permet l'utilisation de la langue cible en société, car d'autres acteurs sociaux en partagent également les éléments constitutifs, en tant que langue première ou seconde (étrangère).

Terme anglais équivalent: Linguistic Language Competence

La compétence langagière pragmatique a trait à la façon d'organiser, de structurer et d'adapter la langue au sein de contextes de discussion caractérisés par des schémas interactionnels et transactionnels.

Terme anglais équivalent: Pragmatic Language Competence

La compétence langagière sociolinguistique se réfère aux connaissances et habiletés à développer par l'apprenant/utilisateur de langues afin d'être en mesure de faire fonctionner la langue au sein de son contexte social propre, et ce, à dessein d'entamer la communication entre l'apprenant et un ou des locuteurs de la langue cible. Il est ainsi question de la façon d'adapter l'utilisation de la langue en fonction des marqueurs de relations sociales, des règles de politesse, des expressions de la sagesse populaire, des différences de registre, de même que du dialecte et de l'accent.

Terme anglais équivalent: Sociolinguistic Language Competence 


\section{Répertoire pluriculturel}

\section{Répertoire plurilingue}

\section{Compréhension plurilingue}

\section{Médiateur interculturel}

Le répertoire pluriculturel regroupe l'ensemble des aspects culturels propres à un apprenant/utilisateur de langues, et se caractérise par ses dimensions dynamique et évolutive. Cet ensemble culturel, personnel et complexe, est pris en compte par les apprenants/utilisateurs de langues dans la réalisation des tâches, souvent de façon inconsciente.

Terme anglais équivalent: Pluricultural Repertoire

Le répertoire plurilingue regroupe l'ensemble des ressources linguistiques propres (langue première, variété dialectale, accents, etc.) à un apprenant/utilisateur de langues, et se caractérise par ses dimensions dynamique et évolutive. Cet ensemble linguistique, personnel et complexe, est pris en compte par les apprenants/utilisateurs de langues dans la réalisation des tâches, souvent de façon inconsciente.

Terme anglais équivalent: Plurilingual Repertoire

La compréhension plurilingue se réfère à la capacité des apprenants/utilisateurs à comprendre, d'une langue à une autre, et même partiellement, le sens relatif à une situation particulière, qu'elle relève d'une activité pédagogique ou d'un événement réel.

Terme anglais équivalent: Plurilingual Comprehension

Le médiateur interculturel possède la capacité, en tant qu'apprenant/utilisateur, d'agir comme intermédiaire, en mobilisant l'ensemble de son répertoire pluriculturel à dessein d'expliquer le sens relatif à une situation particulière (activité pédagogique ou événement réel) à d'autres acteurs, et ce, afin que ceux-ci soient en mesure de comprendre, d'agir et/ou de produire selon le sens voulu dans le contexte de la situation donnée.

Terme anglais équivalent : Intercultural Mediator 